\title{
Formation sequences and roles of multiple deformation twins during the plastic deformation in nanocrystalline fcc metals
}

\author{
Fuping Yuan*, Xiaolei Wu \\ State Key Laboratory of Nonlinear Mechanics, Institute of Mechanics, Chinese Academy of Science, No. 15, North 4th Ring, West Road, Beijing 100190, People's \\ Republic of China
}

\section{A R T I C L E I N F O}

\section{Article history:}

Received 3 April 2013

Received in revised form

6 May 2013

Accepted 10 May 2013

Available online 20 May 2013

Keywords:

Nanocrystalline metals

Multiple twins

Molecular dynamics simulation

Plastic deformation

\begin{abstract}
A B S T R A C T
Large-scale molecular dynamics (MD) simulations were performed to investigate the tensile plastic deformation of nanocrystalline Ag. With increasing tensile strain, formation of SFs, single deformation twins, V-shaped and T-shaped double twins, and 5-fold twins successively starts to play a role on the plastic deformation of nanocrystalline $\mathrm{Ag}$. The direct evidences of the formation sequences for the following two twinning mechanisms are presented in the present study: GB-mediated intersecting mechanism and self-partial-multiplication twinning mechanism. Moreover, the 5-fold twins are found to be formed by a combination of these two mechanisms. The findings in the present study contribute to the understanding of formation mechanisms and roles of multiple twins on the plastic deformation of nanocrystalline fcc metals with low stacking fault energy.
\end{abstract}

(c) 2013 Elsevier B.V. All rights reserved.

\section{Introduction}

Twinning is one of the major deformation mechanisms in nanocrystalline metals and alloys, especially with low stacking fault (SF) energy, and at low deformation temperatures and high strain rates [1-4]. As we know, coarse-grained metals become more difficult to deform by twinning with decreasing grain size. However, nanocrystalline fcc metals could be easier to deform by twinning with decreasing grain size, reaching a maximum twinning probability at a critical grain size, and then become more difficult again when the grain size decreases further [5,6]. Different from those proposed for coarse-grained counterpart, several formation mechanisms for deformation single twins have been observed and proposed in nanocrystalline fcc metals, such as emissons of partial dislocations and random activition of partials (RAP) from grain boundaries (GBs) $[3,4,7,8]$, GB splitting and migration [9-11], and coincidental overlapping of wide SF ribbons $[9,12]$.

Experimentally, multiple twins could be achieved in fcc metals with low/medium SF energy, as evidenced in the samples processed by the newly developed techniques with high strain rates and/or low temperatures, such as the surface mechanical attrition treatment (SMAT) $[13,14]$ and the surface mechanical grinding treatment (SMGT) [15]. For example, the secondary twins could be

\footnotetext{
* Corresponding author. Tel.: +86 10 82544170; fax: +8610 82543977.

E-mail addresses: fpyuan@lnm.imech.ac.cn (F. Yuan), xlwu@imech.ac.cn (X. Wu).
}

generated in the primary twins in $\mathrm{Cu}$ [16], twinning induced plasticity (TWIP) steels [16,17] and 304 stainless steels [18]. Recent experiments and theoretical model have shown that the hierarchically twinned structures with the secondary twins at the nanoscale can be a novel nanostructured design to achieve higher strength and toughness $[16,17,19]$. As classified by the sources of the twinning partials, three type of twinning mechanisms for multiple twins have been proposed for nanocrystalline metals: (1) twinning partials from different GBs meet each other in a confined area inside grain [18-20]; (2) self-partial-multiplication twinning mechanism, in which continuous growth of multiple twins can be sustained through dislocation reactions at the growing twin interfaces or twin boundaries (TBs) [18,21,22]; and (3) rebound mechanism, in which the twinning partials are generated by the rebounding of partials at a GB or TB [23,24]. However, the direct evidences of the formation sequences for these mechanisms are still lacking. Moreover, the roles of SFs, single deformation twins and multiple deformation twins in the different stages of plastic deformation for fcc nanocrystalline metals with low SF energy are still vague. In this regard, large-scale molecular dynamics (MD) simulations were performed in the present study to illustrate the formation sequences and roles of multiple twins during the plastic deformation of nanocrystalline Ag.

\section{Simulation techniques}

The MD simulations were carried out using the Large-scale Atomic/Molecular Massively Parallel Simulator (LAMMPS) code 
a

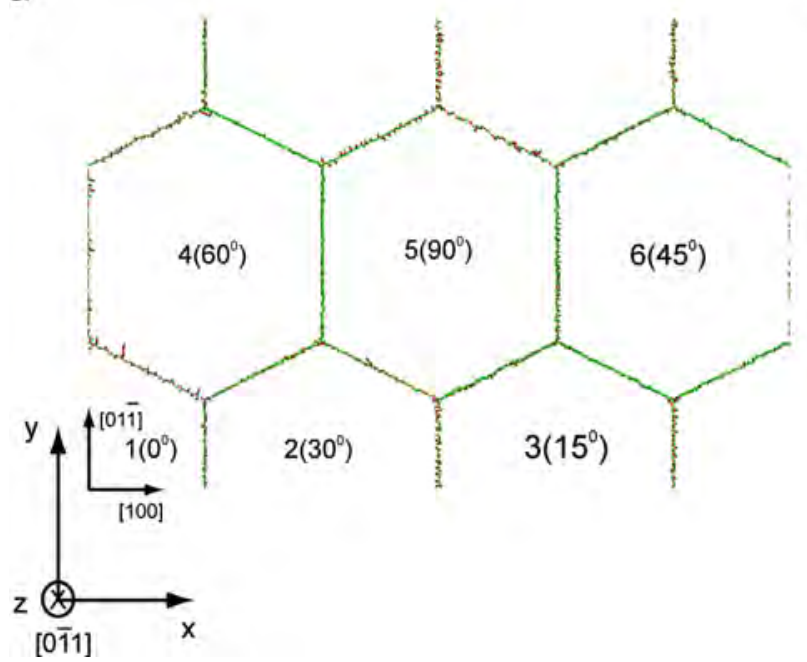

b

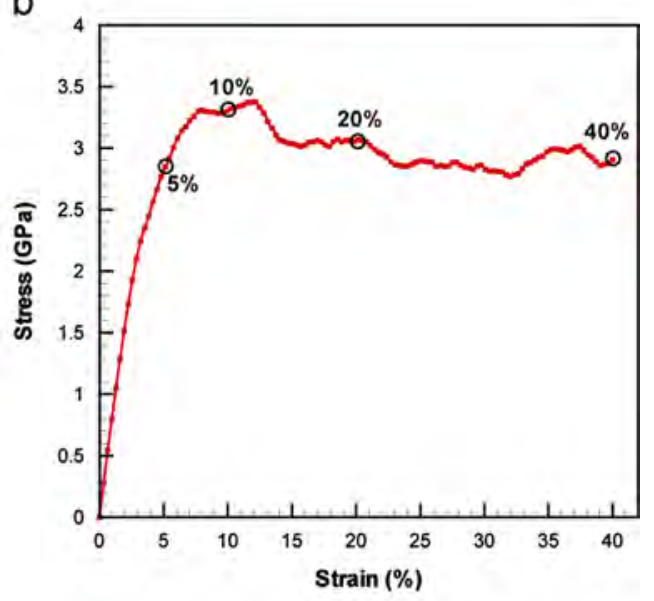

Fig. 1. (a) The relaxed simulation cell with six columnar grains ( $d=60 \mathrm{~nm}$, perfect fcc atoms are not shown in this figure). (b) Simulated tensile stress-strain curve for the simulation cell.

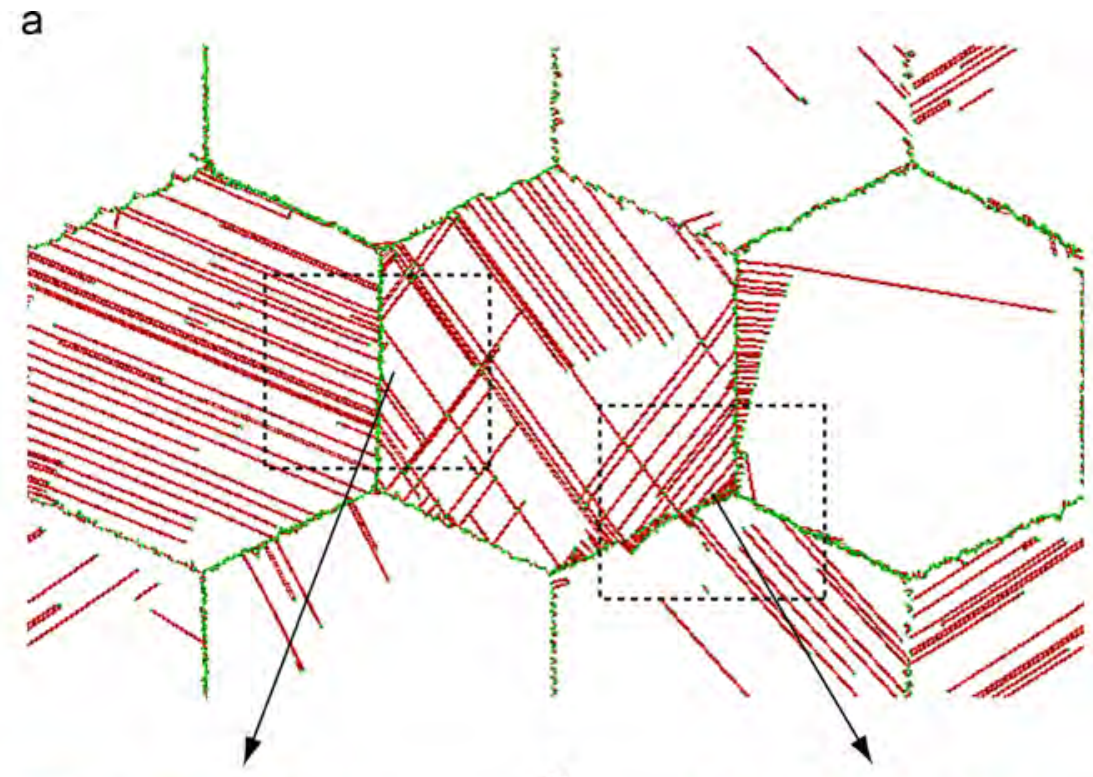

b

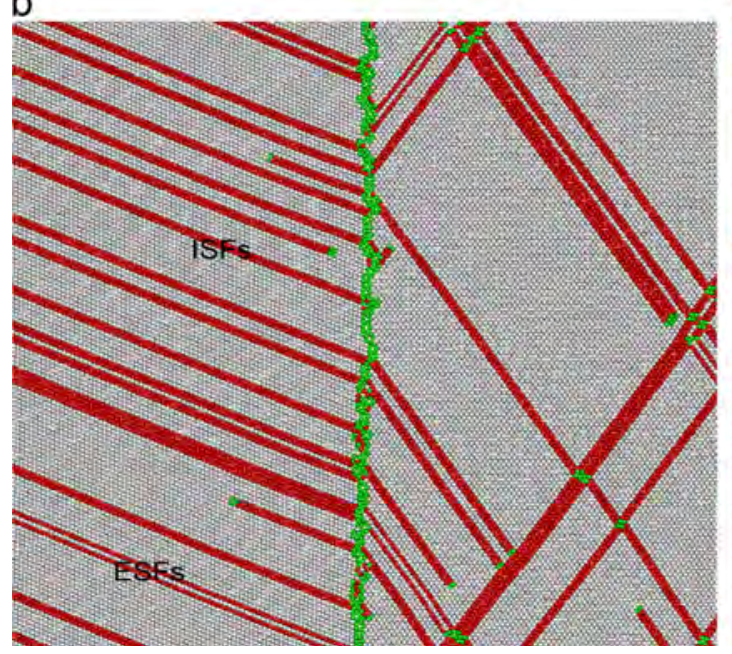

c

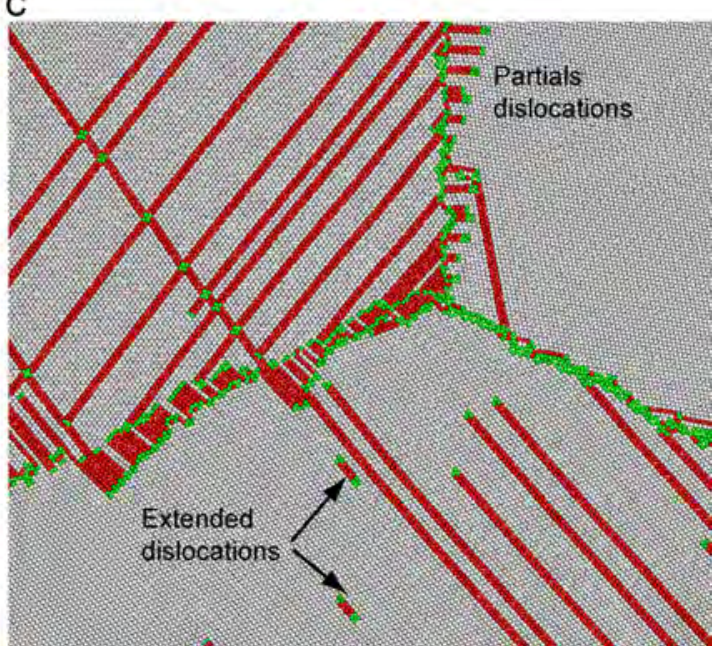

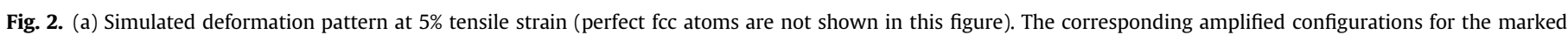
rectangular areas in (a) are shown in (b) and (c). The partial dislocations, intrinsic stacking faults (ISFs), extrinsic stacking faults (ESFs) and extended dislocations are marked in the figure. 
and an Ag EAM potential developed by Williams et al. [25]. Similar to the configuration used by Yamakov [2], quasi 3-dimensional simulations with a columnar grain structure were considered. The thickness direction contains 12 atomic planes, and is along the [01ㅣ direction. The $\langle 011\rangle$ column axis ( $z$-direction) ensures that, following their nucleation, dislocations can glide on either of two $\{111\}$ slip systems in each grain, unimpeded by the 3D-periodic border conditions imposed on the simulation cell [2]. The relaxed simulation cell with six grains is shown in Fig. 1(a), with atoms colored according to common neighbor analysis (CNA) values [26]. Gray color stands for perfect fcc atoms, red color stands for hcp atoms and green color stands for GBs, dislocation core, free surface and other atoms. A single line of hcp atoms represents a TB, two adjacent hcp lines stand for an intrinsic stacking fault (ISF), and two hcp lines with an fcc line between them indicates an extrinsic stacking fault (ESF). The same CNA color coding is used in the following figures. The average grain size $d$ is $60 \mathrm{~nm}$ (the cell has dimensions of $180 \times 120 \times 1.735 \mathrm{~nm}^{3}$, and contain approximately 2,200,000 atoms). Periodic boundary conditions were imposed along all three directions and the tensile loading was along the $x$-direction. The $\langle 100\rangle$ direction in grain 1 is along the $x$-direction, and the marked misorientation angle of each grain is given by the angle between its $\langle 100\rangle$ direction and $x$-direction. The GBs in the simulation cell are all indeed high-angle tilt boundaries. Before tensile loading, the as-created samples were first subjected to energy minimization by the conjugate gradient method, then gradually heated up to the desired temperature in a step-wise fashion, and finally relaxed in the Nose/Hoover isobaric-isothermal ensemble (NPT) under both the pressure 0 bar and the desired temperature $(1 \mathrm{~K})$ for $100 \mathrm{ps}$. After relaxation, a $40 \%$ tensile strain was applied to each sample at a constant strain rate of $5 \times 10^{8} \mathrm{~s}^{-1}$. During the tensile loading, the whole system was kept at a temperature of $1 \mathrm{~K}$ in order to reduce the thermal effect, and the pressures in the $y$ and $z$ directions were kept to zero in order to simulate the uniaxial loading. In MD simulations, the strain rate is typically high ( $>10^{7}$ / s) and the system is typically small (polycrystalline with grain size $<100 \mathrm{~nm}$ ) due to the inherent limitations of MD simulations, which may affect the deformation mechanisms of metals. However, MD simulations have proven to be particularly useful for investigating the plastic deformation mechanism of nanocrystalline metals with a carefully designed model system, in which the transient responses of the system can be examined [3]. The MD simulations should enable uncovering various deformation and microstructural processes (such as formation sequences of multiple deformation twins) in well-designed model systems, something that has proven difficult to achieve in experiments.

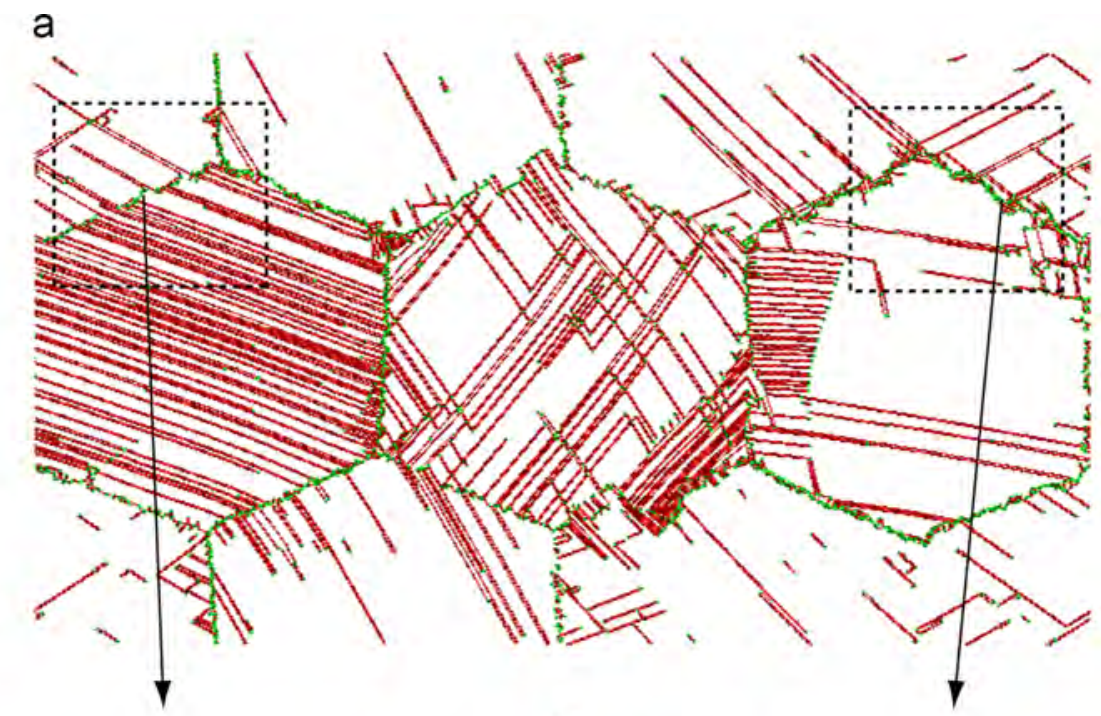

b

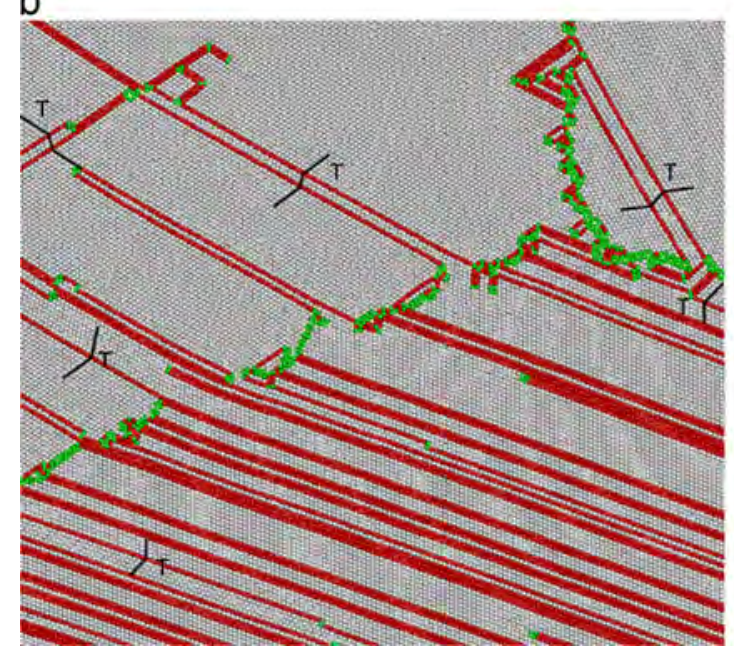

C

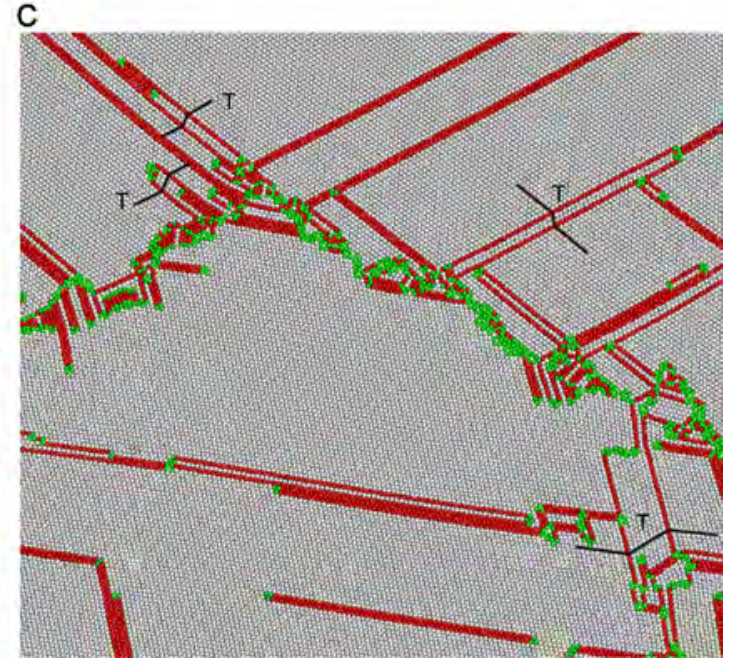

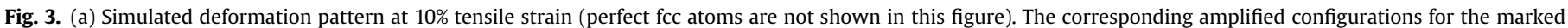
rectangular areas in (a) are shown in (b) and (c). The formed single deformation twins are marked by "T" in the figure. 


\section{Results and discussions}

Fig. 1(b) shows the tensile stress-strain curve for the nanocrystalline $\mathrm{Ag}$ with a grain size of $60 \mathrm{~nm}$. Tensile stress is observed to increase with strain up to a certain peak stress, and then gradually decrease to a steady-state value. After the elastic deformation, the nanocrystalline $\mathrm{Ag}$ shows a strong strain hardening behavior at the early stage of plastic deformation (strain of 3-7.8\%), and then the flow stress reaches the saturation state at the late stage of plastic deformation (strain of 7.8-40\%). The stress-strain curves on finegrained bulk Ag (grain size $1-2 \mu \mathrm{m}$ ) by microshear testing and uniaxial tensile testing can be found in the literature $[27,28]$, although the experimental data on bulk nanocrystalline $\mathrm{Ag}$ (grain size $<100 \mathrm{~nm}$ ) are not available. The experimental data for finegrained bulk $\mathrm{Ag}$ also show a strong strain hardening behavior at the early stage of plastic deformation (plastic strain of $0-10 \%)$, and display a saturate state at large plastic strain [27]. These behaviors are very different from those in coarse-grained $\mathrm{Ag}$, in which the stress-strain curve shows a strain hardening behavior until the very large plastic strain (strain of 200\%) [27]. The strain range for strain hardening behavior should decrease with decreasing grain size of Ag, so the simulated stress-strain curve in the present study reveals a typical deformation behavior of nanocrystalline Ag. In order to investigate the roles of SFs, single deformation twins and multiple deformation twins in the different stages of plastic deformation for fcc nanocrystalline metals with low stacking fault energy, the simulated deformation patterns at tensile strains of $5 \%$, $10 \%, 20 \%$ and $40 \%$ will be presented next.
Fig. 2(a) shows the overall deformation pattern at a tensile strain of 5\%, which is at the onset of plasticity. Figs. 2(b) and 2(c) show the corresponding amplified configurations for the marked rectangular areas in Fig. 2(a). At this stage, the deformation mechanisms are dominated by partial dislocation behaviors and formation of SFs, with a few of extended dislocations inside the grain. In contrast to coarse-grained metals, in which plastic behaviors are accommodated by full dislocations nucleated from Frank-Read sources, partial dislocations nucleated from GBs of nanocrystalline metals travel across the grains on one of the available $\{111\}$ slip planes, until annihilated at another GB and leaving SFs (ISFs and ESFs) behind. Due to the interactions between partial dislocations and SFs, some dislocation networks are also formed inside the nanocrystal grain to accommodate plastic deformation.

Fig. 3(a) shows the overall deformation pattern at a tensile strain of $10 \%$. Figs. 3(b) and 3(c) show the corresponding amplified configurations for the marked rectangular areas in Fig. 3(a). At this stage, the plastic deformation is accommodated by two mechanisms: (1) partial dislocation behaviors and formation of SFs; and (2) formation of single deformation twins. The single deformation twins are generated by successive emissions of twinning partials from GBs at consecutive slip planes. The formation of single deformation twins on one hand provides strong obstacles to severely impede partial dislocation motion for strengthening, and on the other hand increases extra local sites for dislocation accumulation to enhance strain hardening and sustain additional plastic deformation.

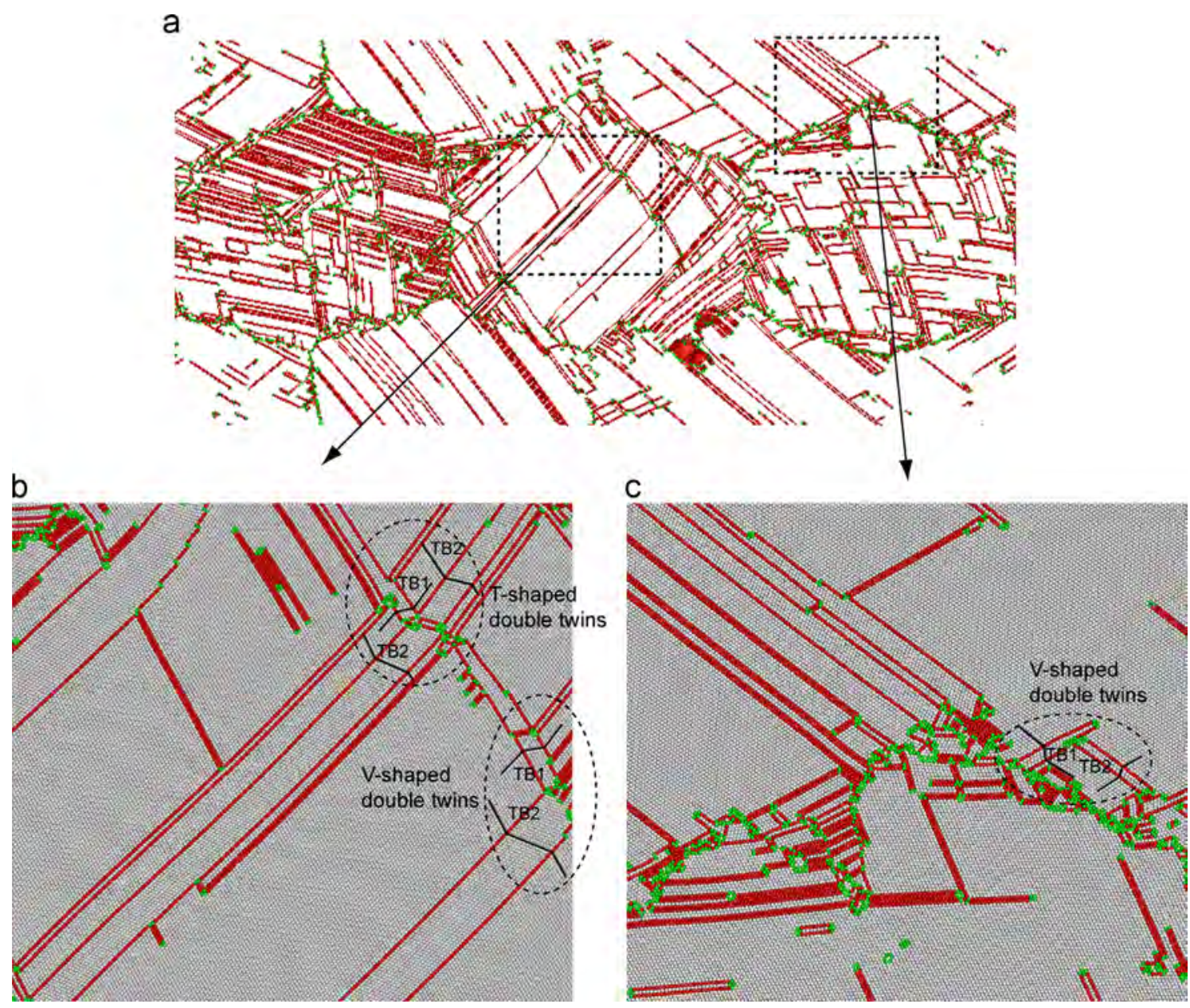

b

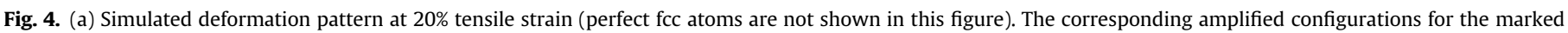
rectangular areas in (a) are shown in (b) and (c). The V-shaped and T-shaped double twins are marked in the figures. 
Fig. 4(a) shows the overall deformation pattern at a tensile strain of $20 \%$. Figs. 4(b) and (c) show the corresponding amplified configurations for the marked rectangular areas in Fig. 4(a). At this stage, besides the two deformation mechanisms mentioned earlier for $5 \%$ tensile strain, a new mechanism starts to play a role in the plastic deformation: formation of V-shaped and T-shaped double twins. One of major issues in the formation of double twins or multiple twins in nanocrystalline fcc metals is the source of twinning partials that are needed on consecutive slip planes for secondary or even high order twins. As classified by the sources of the twinning partials, two common twinning mechanisms of multiple deformation twins have been reported: (1) twinning partials from different GBs meet each other in a confined area inside grain (GB-mediated intersecting mechanism) [18-20]; and (2) self-partial-multiplication twinning mechanism [18,21,22].

Here, we show the direct evidences of formation sequences and provide further confirmation for these two mechanisms, as illustrated in Figs. 5 and 6, respectively. Fig. 5 shows the snapshots illustrating formation of V-shaped double twins by GB-mediated intersecting mechanism. As shown in Fig. 5(a), the nucleation of the first twins is achieved by successive emissions of twinning partials from GB1 at consecutive slip planes. When the plastic deformation is further increased (see Fig. 5(b)), two partial dislocations $\left(b_{1}\right.$ and $b_{2}$ ) are emitted from GB2 and glide toward the TB1 of the first twins along adjacent slip planes, leaving ESFs behind. As shown in Fig. 5(c) and (d), the partial dislocations $b_{1}$ and $b_{2}$ reach and interact with TB1 one by one under the resolved shear stress, and finally are absorbed and stopped at TB1, resulting in formation of $\mathrm{V}$-shaped double twins. The simulated snapshots clearly reveal that the formation mechanism for the $\mathrm{V}$-shaped double twins is the interactions of partial dislocations from different GBs in a confined area (for example, inside the nanocrystal grain in our case).

Fig. 6 shows the snapshots illustrating formation of T-shaped double twins by self-partial-multiplication twinning mechanism. The two dimensional representation of the Thompson tetrahedron is shown in the insert of Fig. 6(a). As shown in Fig. 6(a), a partial dislocation $b_{1}(C \beta)$ emitted from GBs or other sources glides on the (b) plane toward the existing TB1 on the (d) plane, leaving ESF behind. With increasing plastic strain, the partial dislocation $b_{1}$ is stopped at position "P" by TB1. As shown in Fig. 6(b), the partial $b_{1}$ $(C \beta)$ now dissociates into the following dislocations under the applied stress: $C \beta \rightarrow C A+A \delta+\delta \beta$. Although this dislocation reaction is energetically unfavorable, it could have occured with help of the applied shear stress. $A \delta\left(b_{3}\right)$ glides to the lower right to create a one layer step for TB1. The stair-rod dislocation $\delta \beta$ is sessile and cannot move. While $C A$ cross-slips to the next intersection point (toward upper left) and dissociates into two partials on the (b) plane: $C A \rightarrow C \beta+\beta A$. Now, $\beta A\left(\mathrm{~b}_{2}\right)$ glides toward lower left to nucleate a two layer secondary twins on the (b) plane, forming the nucleus of a V-shaped double twins. The partial $C \beta$ can repeat the above dislocation reactions and processes, causing thickening of the secondary twins on the (b) plane, as illustrated in Fig. 6(c). A two layer step will be created for TB1 and the stair-rod a

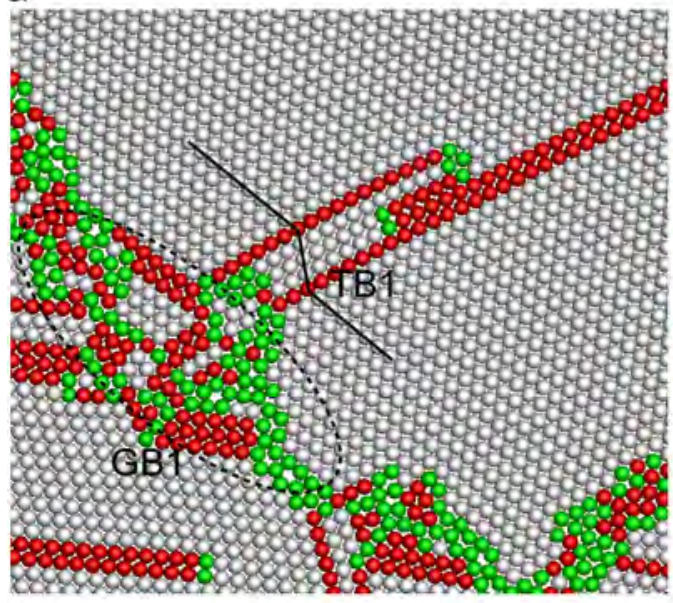

C

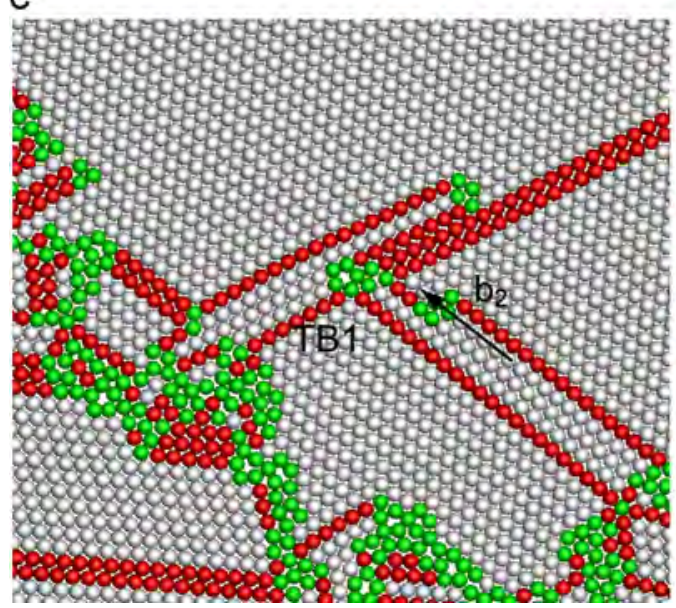

b

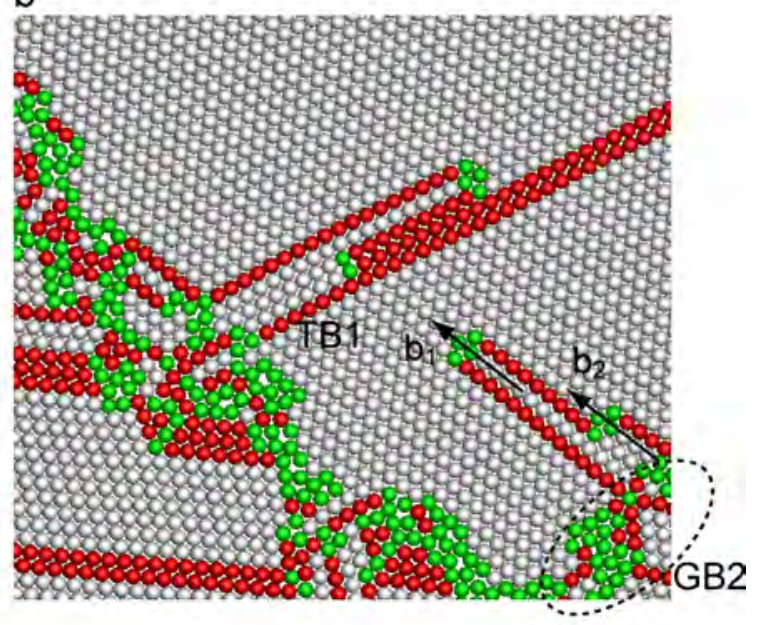

d

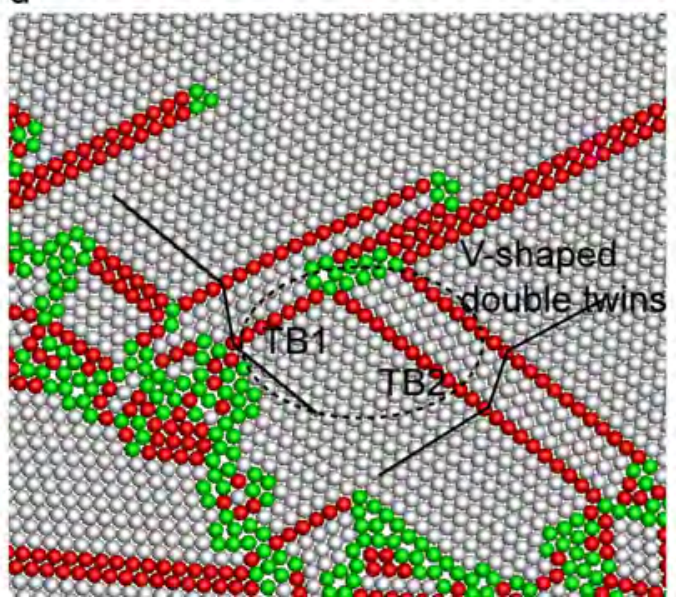

Fig. 5. Simulated snapshots illustrating the formation of V-shaped double twins: at tensile strains of (a) 13.75\%; (b) 14\%; (c) 14.25\%; and (d) 14.5\%. 

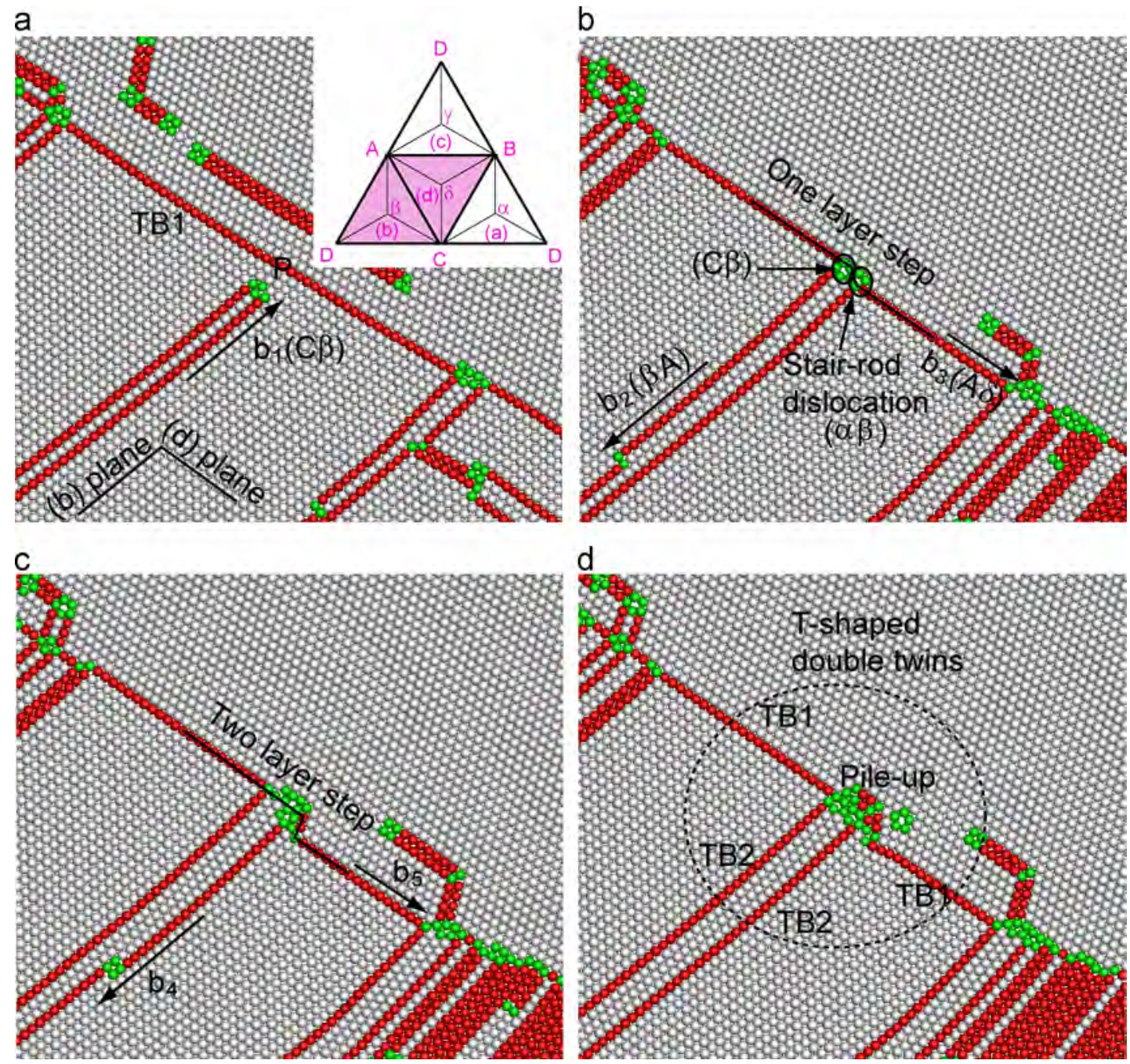

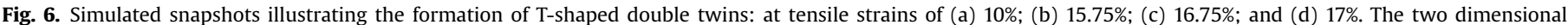
representation of the Thompson tetrahedron is shown in the insert of (a).

dislocation pile-up will be formed at the intersecting position during this thickening process (see Fig. 6(c) and (d)). The simulated snapshots clearly provide the evidence for the similar formation sequences of self-partial-multiplication twinning mechanism, which was proposed for the V-shaped and T-shaped double twins in our previous work [18].

Fig. 7(a) shows the overall deformation pattern at a tensile strain of $40 \%$. Figs. 7(b) and 7(c) show the corresponding amplified configurations for the marked rectangular areas in Fig. 7(a) At this stage, besides the formation of SFs and single deformation twins, the formation of double deformation twins also becomes a dominated plastic deformation behavior (marked by lots of "D" in Figs. 7(b) and 7(c)). Moreover, a few of 5-fold deformation twins are also observed at this large plastic deformation. 5-Fold deformation twins were also reported in nanocrystalline $\mathrm{Al}$ and $\mathrm{Cu}$ recently, and they were proposed to be formed by partial dislocation-mediated sequential twinning [20,29-31]. Fig. 8 shows the snapshots illustrating formation sequences of 5 -fold twins. On the basis of V-shaped double twins (see Fig. 8(a)), two partial dislocations $\left(b_{1}\right.$ and $b_{2}$ ) emitted from GBs or other sources glide toward the intersecting point of the $\mathrm{V}$-shaped double twins, meeting with TB2 and resulting in formation of 3-fold twins (see Fig. 8(b)). By dislocation dissociation and self-thickening, two partial dislocations $\left(b_{3}\right.$ and $\left.b_{4}\right)$ move away from the intersecting position, resulting in formation of 5-fold twins (see Figs. 8(c) and 8 (d)). So the simulated snapshots indicate that the 5-fold twins are formed by a combination of GB-mediated intersecting mechanism and self-thickening twinning mechanism.

\section{Concluding remarks}

In the present study, large-scale MD simulations were performed to illustrate the formation sequences and roles of multiple twins during the plastic deformation of nanocrystalline fcc metals with low stacking fault energy. At the onset of plasticity (5\% of tensile strain), the plastic deformation is completely accommodated by partial dislocation behaviors and formation of SFs. However, the single deformation twins are formed by successive emissions of twinning partials on consecutive slip planes from GBs at larger tensile strain (10\%). With further increasing tensile strain (20\%), the formation of V-shaped and T-shaped double twins starts to play a role on the plastic deformation. At very large tensile strain (40\%), the formation of double deformation twins also becomes a dominated plastic deformation behavior, along with formation of SFs and single deformation twins. Moreover, a few of 5 -fold deformation twins are also observed at tensile strain of $40 \%$. Two common twinning mechanisms were proposed for the formation of multiple twins recently: GB-mediated intersecting mechanism $[18,20]$ and self-partial-multiplication twinning 


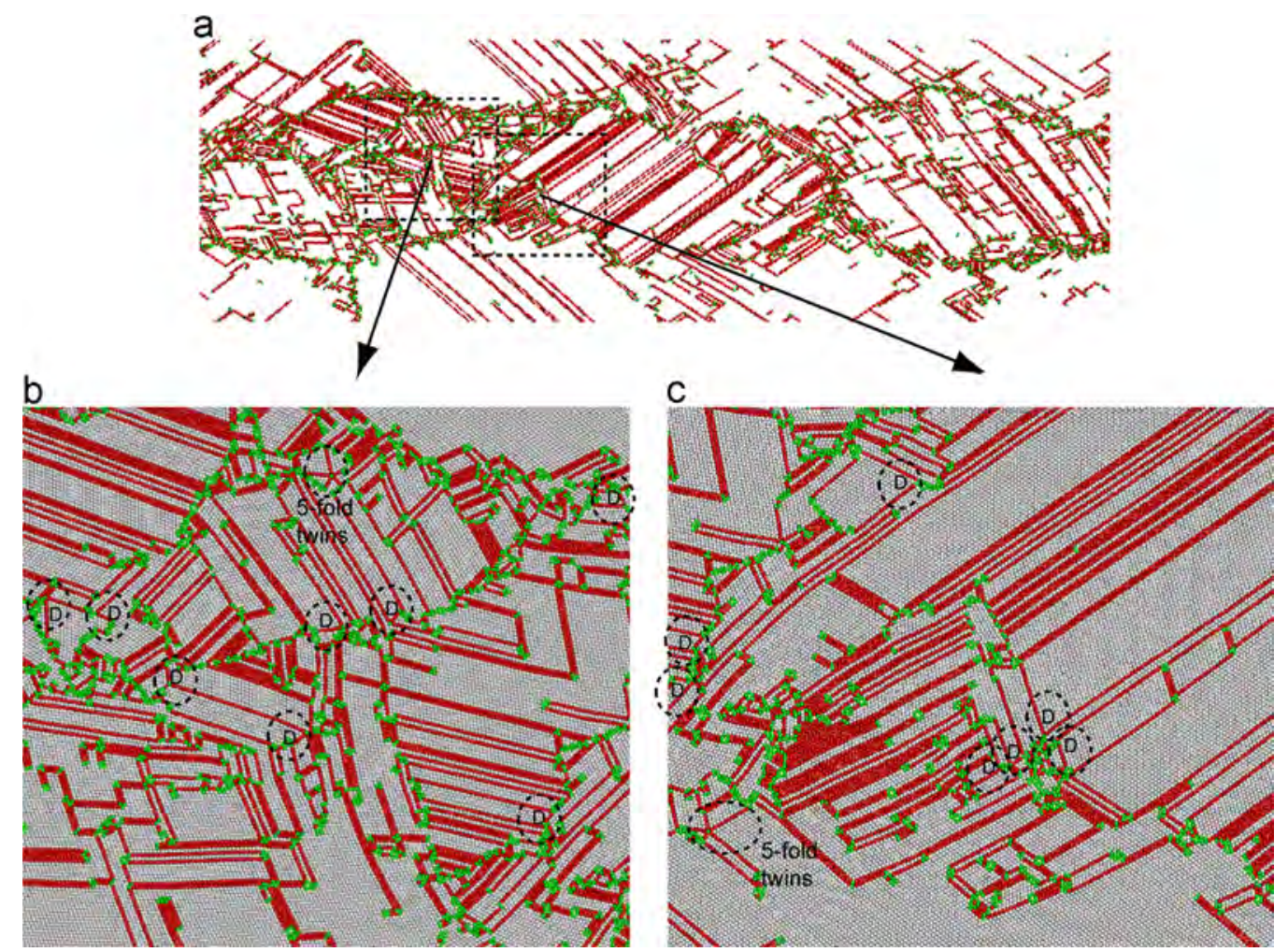

Fig. 7. (a) Simulated deformation pattern at $40 \%$ tensile strain (perfect fcc atoms are not shown in this figure). The corresponding amplified configurations for the marked rectangular areas in (a) are shown in (b) and (c). The double twins are marked by "D", and two 5-fold twins are also marked in the figure.

a

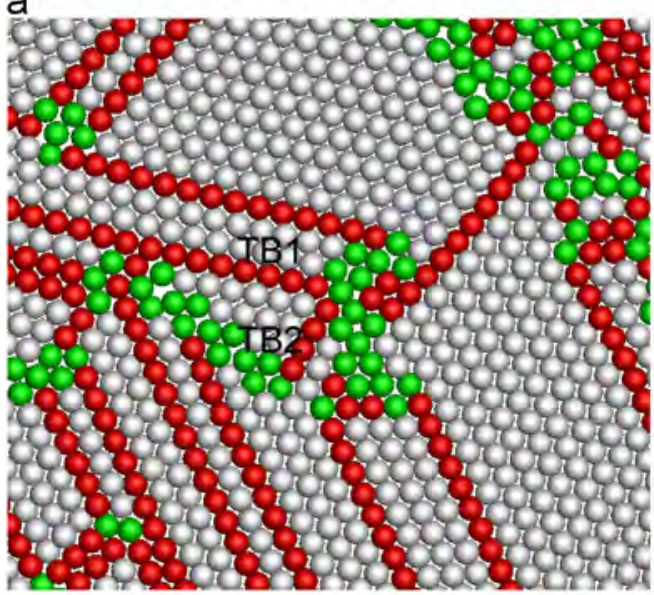

C

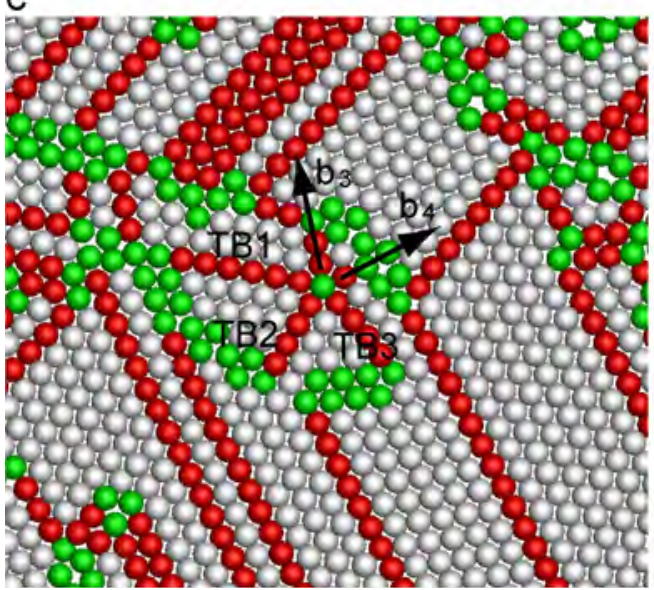

b

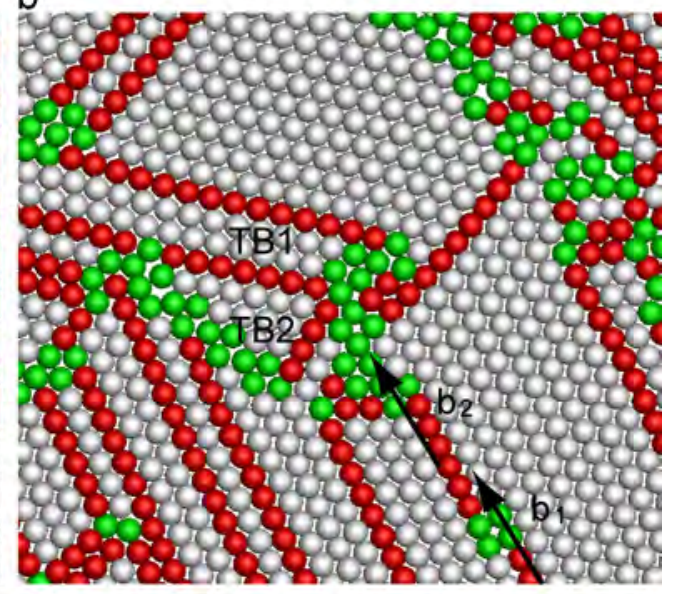

d

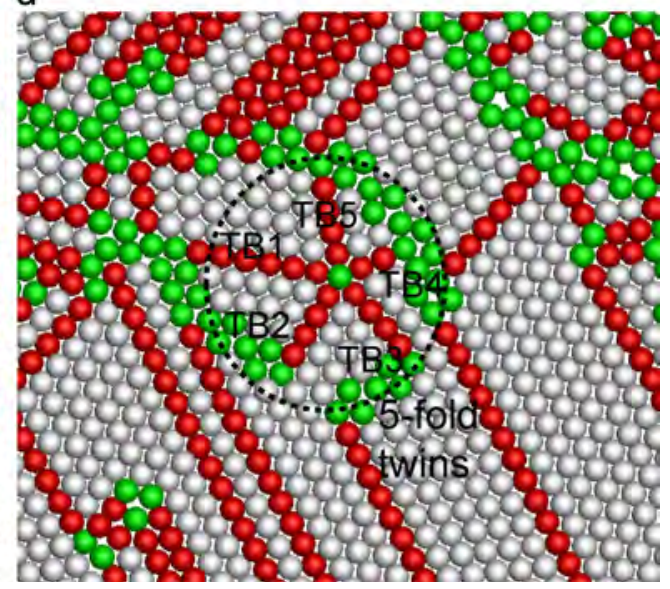

Fig. 8. Simulated snapshots illustrating the formation of 5-fold twins: at tensile strains of (a) 34\%; (b) $35.8 \%$; (c) $35.85 \%$; and (d) $36 \%$. 
mechanism $[18,21,22]$. The direct evidences of the formation sequences for these two mechanisms are presented in the present study. Moreover, the 5-fold twins are found to be formed by a combination of GB-mediated intersecting mechanism and selfthickening twinning mechanism. These multiple twins on one hand carry a great amount of plastic deformation, and on the other hand form hierarchical network obstacles to impede dislocation motion for strengthening. Our findings contribute to the progress of understanding for formation mechanisms of multiple twins in nanocrystalline fcc metals with low stacking fault energy.

\section{Acknowledgments}

The authors would like to acknowledge the financial support of the National Key Basic Research Program of China (Grant nos. 2012 CB932203 and 2012CB937500) and NSFC (Grant nos. 11002151, 11222224, 11072243 and 11021262). The simulations reported were performed at Supercomputing Center of Chinese Academy of Sciences.

\section{References}

[1] J.W. Christian, S. Mahajan, Prog. Mater. Sci. 39 (1995) 1-157.

[2] V. Yamakov, D. Wolf, S.R. Phillpot, A.K. Mukherjee, H. Gleiter, Nat. Mater. (2002) 45-48

[3] D. Wolf, V. Yamakov, S.R. Phillpot, A.K. Mukherjee, H. Gleiter, Acta Mater. 53 (2005) $1-40$.

[4] Y.T. Zhu, X.Z. Liao, X.L. Wu, Prog. Mater. Sci. 57 (2012) 1-62.
[5] X.L. Wu, Y.T. Zhu, Phys. Rev. Lett. 101 (2008) 025503.

[6] J.Y. Zhang, G. Liu, R.H. Wang, J. Li, J. Sun, E. Ma, Phys. Rev. B 81 (2010) 172104

[7] X.L. Wu, Y.T. Zhu, Appl. Phys. Lett. 89 (2006) 031922.

[8] X.L. Wu, X.Z. Liao, S.G. Srinivasan, F. Zhou, E.J. Lavernia, R.Z. Valiev, Y.T. Zhu, Phys. Rev. Lett. 100 (2008) 095701.

[9] V. Yamakov, D. Wolf, S.R. Phillpot, A.K. Mukherjee, H. Gleiter, Nat. Mater. 3 (2004) 43-47.

[10] J. Wang, H.C. Huang, Appl. Phys. Lett. 85 (2004) 5983-5985.

[11] V. Yamakov, D. Wolf, S.R. Phillpot, H. Gleiter, Acta Mater 50 (2002) 5005-5020.

[12] X.Z. Liao, F. Zhou, E.J. Lavernia, S.G. Srinivasan, M.I. Baskes, D.W. He, Y.T. Zhu, Appl. Phys. Lett. 83 (2003) 632-634.

[13] K. Lu, J. Lu, Mater. Sci. Eng. A 375-377 (2004) 38-45.

[14] K. Wang, N.R. Tao, G. Liu, J. Lu, K. Lu, Acta Mater. 54 (2006) 5281-5291.

[15] T.H. Fang, W.L. Li, N.R. Tao, K. Lu, Science 331 (2011) 1587-1590.

[16] N.R. Tao, K. Lu, Scr. Mater. 60 (2009) 1039-1043.

[17] H.N. Kou, Ph.D. dissertation, The Hong Kong Polytechnic University, 2011.

[18] Y.T. Zhu, J. Narayan, J.P. Hirth, S. Mahajan, X.L. Wu, X.Z. Liao, Acta Mater. 57 (2009) 3763-3770

[19] L.L. Zhu, H.N. Kou, J. Lu, Appl. Phys Lett. 101 (2012) 081906

[20] A.J. Cao, Y.G. Wei, Appl. Phys Lett. 89 (2006) 041919.

[21] J. Narayan, Y.T. Zhu, Appl. Phys. Lett. 92 (2008) 151908.

[22] X.L. Wu, J. Narayan, Y.T. Zhu, Appl. Phys. Lett. 93 (2008) 031910.

[23] S.A. Dregia, J.P. Hirth, J. Appl. Phys. 69 (1991) 2169-2175.

[24] C.H. Henager Jr., R.G. Hoagland, Scr. Mater. 50 (2004) 701-705.

[25] P.L. Williams, Y. Mishin, J.C. Hamilton, Modell. Simul. Mater. Sci. Eng. 14 (2006) 817-833.

[26] H. Tsuzuki, P.S. Branicio, J.P. Rino, Comput. Phys. Commun. 177 (2007) 518-523.

[27] M.E. Kassner, P. Geantil, A.M. Hodge, R.S. Rosen, Scr. Mater. 61 (2009) 721-724

[28] K.J.H. Al-Fadhalah, C. Li, A.J. Beaudoin, D.A. Korzekwa, I.M. Robertson, Acta Mater. 56 (2008) 5764-5774.

[29] X.Z. Liao, J.Y. Huang, Y.T. Zhu, F. Zhou, E.J. Lavernia, Philos. Mag. 83 (2003) 3065-3075.

[30] Y.T. Zhu, X.Z. Liao, R.Z. Valiev, Appl. Phys. Lett. 86 (2005) 103112.

[31] J.Y. Huang, Y.K. Wu, H.Q. Ye, Acta Mater. 44 (1996) 1211-1221. 